\title{
Hybrid Analog-Digital Beam Training for mmWave Systems with Low-Resolution RF Phase Shifters
}

\author{
Danilo De Donno, Joan Palacios Beltrán, Domenico Giustiniano, and Joerg Widmer \\ IMDEA Networks Institute \\ Madrid, Spain \\ Emails: name.surname@imdea.org
}

\begin{abstract}
Millimeter wave (mmWave) wireless technologies are expected to exploit large-scale multiple-input multiple-output and adaptive antenna arrays at both the transmitter and receiver to deal with unfavorable radio propagation and realize sufficient link margin. However, the high cost and power consumption of mmWave radio components prohibit the use of fully-digital precoding/combining architectures, which incurs one dedicated RF chain per antenna element. This paper proposes a practical design of multi-beamwidth codebook exploiting hybrid analogdigital architectures with a number of RF chains much lower than the number of antenna elements and 2-bit RF phase shifters. The proposed solution relies on the orthogonal matching pursuit algorithm enhanced by a dynamic dictionary learning mechanism. Simulation results show that the designed hybrid codebooks are able to shape beam patterns very close to those attained by a fully-digital beamforming architecture. Furthermore, when leveraged in the framework of an adaptive, multiresolution beam training protocol, our hybrid codebooks are able to estimate the most promising angle-of-departure and angle-ofarrival directions with extreme accuracy, yet requiring lower complexity hardware compared to the state of art.
\end{abstract}

\section{INTRODUCTION}

The exponential growth of mobile data traffic and the need to support higher user data rates has caused a bandwidth shortage at frequencies below $6 \mathrm{GHz}$, known as the spectrum crunch problem. As part of the future fifth-generation (5G) networks, millimeter-wave (mmWave) technology is an important candidate to provide the much needed bandwidth to solve the wireless spectrum crunch and support $\mathrm{Gb} / \mathrm{s}$ data rates [1], [2].

Communication systems based on mmWave frequencies are expected to largely differ from those operating at frequencies below $6 \mathrm{GHz}$. First, due to the higher propagation loss and unfavorable atmospheric absorption, data transmission over relatively long distances represents a serious challenge at mmWaves. Second, the short wavelength allows more antenna elements to be integrated into devices and base stations operating in this band, thus enabling the implementation of largescale multiple-input multiple-output (MIMO) and adaptive antenna arrays to improve the link budget.

The high cost, power consumption, and complexity of mmWave mixed signal components prohibit the use of fullydigital beamforming architectures (i.e., with one dedicated Radio Frequency (RF) chain per antenna element) conventionally adopted in sub-6 GHz MIMO systems [3]. On the other hand, analog beamforming solutions found in current mmWave RF integrated circuit (RFIC) designs are sub-optimal due to the constant magnitude and low-resolution of RF phase shifters.

Designing efficient, low-overhead beamforming training protocols is another crucial aspect at mmWaves [4]. In fact, the beam training phase requires that mmWave device pairs exchange several training packets at a set of predefined directions in order to determine the optimal antenna patterns for subsequent data transmissions. As a viable option to provide a trade-off between performance and cost, hybrid architectures with analog-digital beamforming have gained considerable attention, thanks to the fewer RF chains needed compared to pure digital beamforming architectures. The design space of algorithms presented in the literature for hybrid architectures has until very recently made the unrealistic assumption of considering RF phase shifters with large number of quantization bits or even unconstrained RF phase shifters. In [5], a codebook design algorithm is developed under the assumption that hybrid analog-digital beamforming is used only at the base station while the mobile station is equipped with a single antenna. The hybrid codebook is designed by minimizing the mean square error (MSE) between the code vector's beam pattern and its corresponding ideal beam pattern. The approximation of MSE minimization is accomplished by the orthogonal matching pursuit (OMP) algorithm with unconstrained digitally-controlled RF phase shifters (i.e., with infinite number of quantization bits). A similar OMP-based approach with unconstrained RF phase shifters is discussed also in [6]. A hybrid analog-digital multi-resolution codebook relying on beamforming vectors with different beamwidths and gains is presented, for the first time, in [7]. The ability to generate beams with various beamwidths makes such codebook particularly attractive for the design of adaptive, low-overhead beam training protocols. The approach in [7] assumes that RF phase shifters with large number of quantization bits are available at mmWaves. However, the current state of silicon technologies makes challenging and even impractical the design of RF phase shifters with high phase shift resolution [8]. Very recently, in [9] and [10], RF phase shifters with fewer quantization bits have been exploited for the design of hybrid analog-digital codebooks consisting, however, only of narrow beams with fixed beamwidth.

In this paper, we propose a codebook design for mmWave systems with a hybrid analog-digital transceiver architecture and practical constraints in the realization of the transceiver. 
Our contributions are listed as follows:

- we resort to a classical antenna array design and a fullydigital beamforming architecture (i.e. with one RF chain per antenna element) to define the baseline codebook consisting of almost ideal beam patterns, easily configurable in terms of both beamwidth and steering direction;

- we formulate an optimization problem to approximate the baseline fully-digital codebook by means of a hybrid architecture requiring a number of RF chains much lower than the number of antenna elements and only 2-bit RF phase shifters;

- we implement a variant of the classical OMP algorithm to efficiently solve the optimization problem. The proposed algorithm relies on a dynamic dictionary learning (DDL) mechanism which allows to synthesize beam patterns displaying reduced sidelobe level, excellent flatness over the covered sector, and limited overlap with adjacent beams;

- we adopt a realistic channel model capturing the scattering nature of mmWave wireless communications and leverage the designed hybrid codebooks in the framework of an adaptive, multi-resolution beam training protocol.

Compared to state-of-art algorithms which are very sensitive to the number of RF chains and require unconstrained RF phase shifters [5], [6], or RF phase shifters with 6-7 bits resolution [7], our solution provides significantly better performance with lower complexity hardware.

\section{SYSTEM MODEL}

We consider the mmWave system shown in Fig. 1. A base station (BS) equipped with a uniform linear array (ULA) of $M_{\mathrm{BS}}$ isotropic radiators and $N_{\mathrm{RF}-\mathrm{BS}} \mathrm{RF}$ transceiver chains is assumed to communicate with a single mobile station (MS) equipped with a ULA of $M_{\mathrm{MS}}$ isotropic radiators and $N_{\mathrm{RF}-\mathrm{MS}}$ $\mathrm{RF}$ transceiver chains. In this paper, we focus on the beam training phase during which $\mathrm{BS}$ and MS communicate via a single stream of data.

In the hybrid analog-digital approach, the BS applies an $N_{\text {RF-BS }} \times 1$ digital baseband precoder $\mathbf{p}_{\mathrm{BB}}$ followed by an $M_{\mathrm{BS}} \times N_{\mathrm{RF}-\mathrm{BS}} \mathrm{RF}$ precoder, $\mathbf{P}_{\mathrm{RF}}$, to the discrete-time transmitted symbol $s(t)$. In the same way, at the MS, an $M_{\mathrm{MS}} \times N_{\mathrm{RF}-\mathrm{MS}}$ $\mathrm{RF}$ combiner $\mathbf{C}_{\mathrm{RF}}$ followed by a $N_{\mathrm{RF}-\mathrm{MS}} \times 1$ digital baseband combiner $\mathbf{c}_{\mathrm{BB}}$ is used to process the discrete-time received signal:

$$
y(t)=\sqrt{\rho} \mathbf{c}^{H} \mathbf{H p} s(t)+\mathbf{c}^{H} n(t)
$$

where $\mathbf{c}=\mathbf{C}_{\mathrm{RF}} \mathbf{c}_{\mathrm{BB}}$ (dimensions $\left.M_{\mathrm{MS}} \times 1\right), \mathbf{p}=\mathbf{P}_{\mathrm{RF}} \mathbf{p}_{\mathrm{BB}}$ (dimensions $M_{\mathrm{BS}} \times 1$ ), $\rho$ is the average received power, $\mathbf{H}$ is the $M_{\mathrm{MS}} \times M_{\mathrm{BS}}$ mmWave channel matrix, and $n(t) \sim C \mathcal{C N}\left(0, \sigma^{2}\right)$ is the complex white Gaussian noise.

Similar to [6]-[9], we consider a geometric channel model with $L$ paths expressed as:

$$
\mathbf{H}=\sqrt{\frac{M_{\mathrm{BS}} M_{\mathrm{MS}}}{L}} \sum_{l=1}^{L} \alpha_{l} \mathbf{a}_{\mathrm{MS}}\left(\phi_{\mathrm{MS}, l}\right) \mathbf{a}_{\mathrm{BS}}^{H}\left(\phi_{\mathrm{BS}, l}\right)
$$

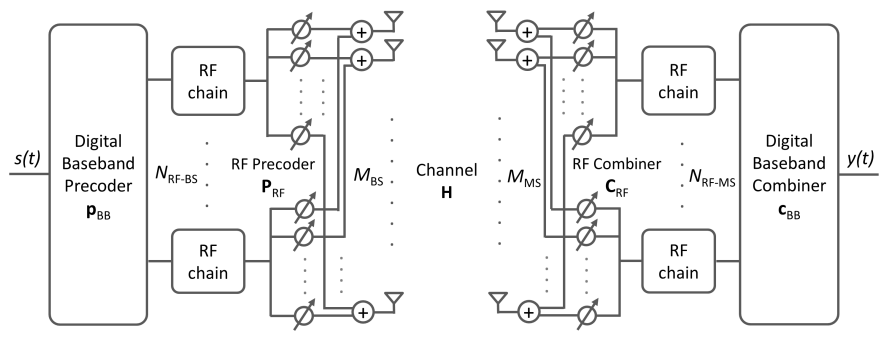

Fig. 1. Overview of the BS/MS mmWave transceiver architecture for hybrid analog-digital beamforming.

where $\alpha_{l} \sim \mathcal{C N}(0,1)$ is the complex gain of the $l^{\text {th }}$ path and $\mathbf{a}_{\mathrm{MS} \text { (BS) }}\left(\phi_{\mathrm{MS}(\mathrm{BS}), l}\right)$ is the ULA steering vector at the MS (BS) which can be written as:

$$
\begin{aligned}
\mathbf{a}_{\mathrm{MS}(\mathrm{BS})}\left(\phi_{\mathrm{MS}(\mathrm{BS}), l}\right)= & \frac{1}{\sqrt{M_{\mathrm{MS}(\mathrm{BS})}}}\left(1, \mathrm{e}^{j \frac{2 \pi}{\lambda} d \sin \left(\phi_{\mathrm{MS}(\mathrm{BS}), l}\right)}, \ldots\right. \\
& \left.\ldots, \mathrm{e}^{j\left(M_{\mathrm{MS}(\mathrm{BS})}-1\right) \frac{2 \pi}{\lambda} d \sin \left(\phi_{\mathrm{MS}(\mathrm{BS}), l}\right)}\right)^{H}
\end{aligned}
$$

where $\phi_{\mathrm{MS}}(\mathrm{BS}), l \in[0,2 \pi]$ is the $l^{\text {th }}$ path's azimuth angles of departure/arrival (AoDs/AoAs) of the BS (MS), $\lambda$ is the signal wavelength, and $d$ is the antenna element spacing.

Our objective is to design a multi-beamwidth codebook based on the mmWave hybrid architecture in Fig. 1] and assuming RF phase shifters with few quantization bits. Such a codebook can be leveraged in the framework of low-overhead beam training with the goal of estimating the best AoD/AoA as discussed in [7].

\section{BEAM TRAINING ALGORITHM AND CODEBOOK DESIGN}

In this section, we first revisit the beam training algorithm proposed in [7] where the beamforming vectors are adaptively configured at both the BS and MS sides based on the bisection concept. Although the algorithm assumes the availability of a feedback channel between the BS and MS, such requirement can be easily relaxed by using the ping-pong approach described in [11].

The algorithm starts by dividing the $[0,2 \pi]$ azimuthal domain into two partitions and by designing the best hybrid analog-digital precoders and combiners to sense those partitions. Formally, at the first stage of the algorithm, the BS employs two beamforming vectors, namely $\mathbf{p}_{1,1}$ and $\mathbf{p}_{1,2}$, to transmit the beacon signal at two successive time slots. At the same time, the MS employs two measurement vectors, namely $\mathbf{c}_{1,1}$ and $\mathbf{c}_{1,2}$, at two successive instants to detect the beacon signal transmitted by the BS over each of the beamforming vectors. The MS compares the signal-to-noise ratio (SNR) of the received beacon signals to determine the one with the maximum SNR. This translates into selecting the partitions which are highly likely to contain the most dominant AoD/AoA combination of the mmWave channel. The MS then communicates the search results to the BS to prepare for the later stages where the selected partitions are further divided into smaller subsets as shown in Fig. 2 until the AoD/AoA pair 


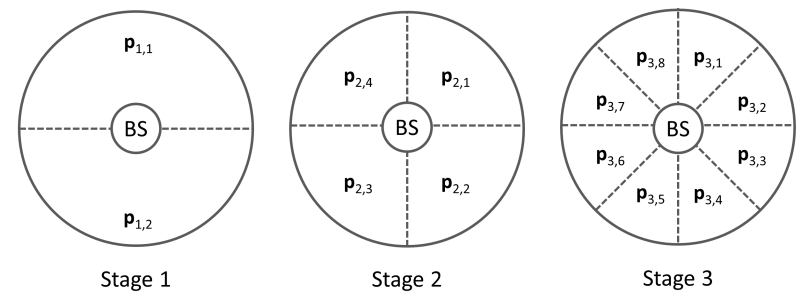

Fig. 2. Structure of the multi-beamwidth beamforming vectors at the BS and relative azimuthal partitions covered by the beams in the first three stages of the beam training protocol.

is estimated with the desired resolution. In case of a reciprocal channel, the number of adaptive stages needed to estimate the most dominant AoD/AoA with angular resolution $2 \pi / N$ is $\mathrm{S}=\log _{2} N$. In case of a non-reciprocal channel, instead, the beam training algorithm is repeated with $\mathbf{H}$ replaced by the uplink channel and the roles of the BS and MS reversed.

The problem of designing the best beamforming (measurement) vectors at the BS (MS) side can be translated into the one of designing, at each stage of the adaptive training algorithm, beam patterns able to optimally cover the current angular sectors shown in Fig. 2 and to minimize the AoD/AoA estimation errors. The main characteristics of the desired beam patterns are:

- limited overlap of adjacent beam patterns;

- flat-top shape over the covered angular region;

- limited number and intensity of side lobes.

In the next subsection, we discuss the baseline beam patterns that the hybrid analog-digital codebook design presented in $\$$ III-B will attempt to accurately represent.

\section{A. Baseline beam patterns}

Baseline beam patters can be synthesized by exploiting a fully-digital beamforming architecture where the availability of a dedicated RF chain for each antenna element enables precise control of both phase and amplitude of the mmWave signals. We choose the sector beam array design based on the Fourier Series Method with Kaiser windowing (FSM$\mathrm{KW})$ to synthesize angular patterns confined to a desired angular region [12, Chapter 21, pp. 946-949]. Compared to other windows such as Hamming, Blackman, etc., the Kaiser window has more design flexibility since the trade-off between the main lobe width and the sidelobe ripple amplitude can be accurately set by adjusting some window parameters.

In this subsection, we focus on the codebook design at the BS, but similar considerations hold also for the MS. Given a desired beam pattern according to the specification mask in Fig. 3, the FSM-KW calculates the baseline array weights for a ULA with $M_{\mathrm{BS}}$ half-wavelength spaced elements as:

$$
\mathbf{p}_{\mathrm{bsl}}(m)=\mathbf{w}(m) e^{-j \beta \psi_{0}} \frac{\sin \left(\psi_{b} \beta\right)}{\pi \beta}, m=0,1, \ldots, M_{\mathrm{BS}}-1
$$

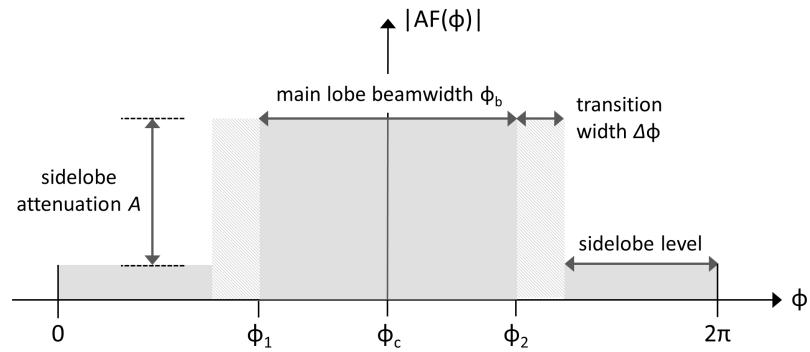

Fig. 3. Magnitude of the array factor used as beam pattern specification mask for designing the FSM-KW array weights.

where $\mathbf{w}(m)$ are the samples of the Kaiser window, $\beta=m-$ $M_{\mathrm{BS}} / 2$, and $\psi_{0}$ and $\psi_{b}$ are defined as follows:

$$
\begin{aligned}
& \psi_{0}=\frac{\pi}{2}\left(\cos \phi_{1}+\cos \phi_{2}\right) \\
& \psi_{b}=\pi \sin \left(\phi_{c}\right) \sin \left(\frac{\phi_{b}}{2}\right)+\frac{\pi D}{M_{\mathrm{BS}}-1}
\end{aligned}
$$

From the sidelobe attenuation $A$ (in $\mathrm{dB}$ ), the $D$-factor and shape parameter $\gamma$ of the Kaiser window can be calculated as:

$$
D= \begin{cases}\frac{A-7.95}{14.36}, & \text { if } A>21 \\ 0.922, & \text { if } A \leq 21\end{cases}
$$

$$
\gamma= \begin{cases}0.11(A-8.7), & \text { if } A \geq 50 \\ 0.58(A-21)^{0.4}+0.079(A-21), & \text { if } 21<A<50 \\ 0, & \text { if } A \leq 21\end{cases}
$$

Finally, the samples of the Kaiser window are given by:

$$
\mathbf{w}(m)=\frac{I_{0}\left(\gamma \sqrt{1-\frac{4 \beta^{2}}{M_{\mathrm{BS}}^{2}}}\right)}{I_{0}(\gamma)}
$$

where $I_{0}(\cdot)$ is the modified Bessel function of first kind and zero-th order. More details about the FSM-KW formulation can be found in [12, Chapter 21, pp. 946-949].

\section{B. Hybrid analog-digital beam patterns}

The codebook design defined in the previous subsection assumes the availability of a fully-digital beamforming architecture with a dedicated RF chain for each antenna element. However, such an architecture is not practical at mmWave frequencies due to the high cost, complexity, and power consumption of the RF chains. In this subsection, we present a practical codebook design that approximates the baseline fullydigital design with a hybrid analog-digital architecture. Our codebook design requires a number of RF chains much lower than the number of antenna elements, and RF phase shifters with just four phase values $(0, \pm \pi / 2, \pi)$ without amplitude adjustment.

As in the previous subsection, we focus on the codebook design at the BS, but similar considerations hold also for the MS. At each stage of the adaptive beam training protocol, i.e., for each combination of steering direction and beamwidth, the hybrid analog-digital codebook design problem consists of 
finding the optimal RF (baseband) precoder $\mathbf{P}_{\mathrm{RF}}^{\mathrm{opt}}\left(\mathbf{p}_{\mathrm{BB}}^{\mathrm{opt}}\right)$ such that:

$$
\begin{gathered}
\left(\mathbf{P}_{\mathrm{RF}}^{\mathrm{opt}}, \mathbf{p}_{\mathrm{BB}}^{\mathrm{opt}}\right)=\underset{\mathbf{P}_{\mathrm{RF}}, \mathbf{p}_{\mathrm{BB}}}{\arg \min }\left\|\mathbf{p}_{\mathrm{bsl}}-\mathbf{P}_{\mathrm{RF}} \mathbf{p}_{\mathrm{BB}}\right\|_{2} \\
\text { s.t. } \quad\left[\mathbf{P}_{\mathrm{RF}}\right]_{:, i} \in\left\{\left[\mathbf{A}_{\mathrm{can}}\right]_{:, \ell} \mid 1 \leq \ell \leq N_{\mathrm{can}}\right\} \\
\quad i=1,2, \ldots, N_{\mathrm{RF}-\mathrm{BS}} \\
\quad\left\|\mathbf{P}_{\mathrm{RF}} \mathbf{p}_{\mathrm{BB}}\right\|_{2}^{2}=1
\end{gathered}
$$

where $\mathbf{p}_{\text {bsl }}$ is the baseline beam pattern to approximate calculated as in Eq. (4). The finite set of possible analog beamforming vectors is encompassed by the $M_{\mathrm{BS}} \times N_{\text {can }}$ matrix $\mathbf{A}_{\text {can }}$ which is also referred to as dictionary in the sequel.

In this paper, we rely on the analog beamforming codebook design in [13] to define our dictionary. Specifically, when $N_{\text {can }}=M_{\mathrm{BS}}$, the matrix $\mathbf{A}_{\text {can }}$ can be defined as:

$$
\begin{aligned}
& {\left[\mathbf{A}_{\mathrm{can}}\right]_{m, \ell}=j^{\text {floor }\left[\frac{(m-1) \times \bmod \left[(\ell-1)+\left(M_{\mathrm{BS}} / 2\right), M_{\mathrm{BS}}\right]}{M_{\mathrm{BS}} / 4}\right],}} \\
& m=1,2, \ldots, M_{\mathrm{BS}} ; \ell=1,2, \ldots, M_{\mathrm{BS}}
\end{aligned}
$$

where the function floor[-] returns the biggest integer smaller than or equal to its argument and $\bmod [\cdot, \cdot]$ is the modulo operation. Using Eq. (9) ensures that the elements of $\mathbf{P}_{\mathrm{RF}}$ have unitary modulus and only four possible phase values, namely $(0, \pm \pi / 2, \pi)$, which allows for the use of RF phase shifters with just two quantization bits.

If we remove the constraint $\left\|\mathbf{P}_{\mathrm{RF}} \mathbf{p}_{\mathrm{BB}}\right\|_{2}^{2}=1$ from Eq. (8), the optimization problem is equivalent to approximate $\mathbf{p}_{\mathrm{bsl}}$ as:

$$
\mathbf{p}_{\mathrm{bsl}} \approx \sum_{i=1}^{N_{\mathrm{BS}-\mathrm{RF}}}\left[\mathbf{P}_{\mathrm{RF}}\right]_{:, i} \mathbf{p}_{\mathrm{BB}}(i)
$$

where $\left[\mathbf{P}_{\mathrm{RF}}\right]_{:, i}$ is restricted to be a column of $\mathbf{A}_{\mathrm{can}}$. Such a problem can be easily solved using OMP algorithms with $N_{\text {BS-RF }}$ iterations as in [5]-[7]. At each iteration, the basic OMP algorithm selects the dictionary column (i.e., a column from $\mathbf{A}_{\text {can }}$ ) along which the current beamforming vector (residual) has the maximum projection. Once the column is selected, its contribution is removed from the dictionary by orthogonally projecting it out - concretely, this means setting all the elements of the selected column to zero. The process continues until all the $N_{\mathrm{BS}-\mathrm{RF}}$ beamforming vectors making up $\mathbf{P}_{\mathrm{RF}}$ have been selected.

It is clear that nullifying some dictionary columns makes the basic OMP algorithm dealing with a less informative dictionary at each iteration. This translates into the selection of sub-optimal dictionary columns as the algorithm moves forward. In order to overcome such a limitation, we propose an OMP algorithm with a dynamic dictionary learning mechanism (DDL-OMP) which, at each iteration, replaces the column projected out from $\mathbf{A}_{\text {can }}$ with another vector that is close to the residual. In this way, the original dictionary is progressively updated with columns which are highly likely candidates to approximate the baseline beam pattern.

The pseudo-code of the proposed DDL-OMP is outlined in the Algorithm 1. It takes as input parameter the baseline precoder to approximate $\mathbf{p}_{\mathrm{bsl}}$. Then, it defines an extended matrix $\mathbf{A}_{\text {can }}$ obtained by adding a column vector $\mathcal{S}\left(\mathbf{p}_{\text {bsl }}\right)$ to the matrix in Eq. (9). We denote $S(\mathbf{v})$ an operator that maps the vector $\mathbf{v}$ into one close vector attainable with 2-bit RF phase shifters. In other words, $S(\mathbf{v})$ maps each component $\mathbf{v}(\ell)$ to the nearest value $\mathrm{e}^{j \phi_{\ell}}$, being $\phi_{\ell} \in\left\{-\frac{\pi}{2}, 0, \frac{\pi}{2}, \pi\right\}$. The algorithm proceeds by finding the vector $\Phi$ along which the baseline precoder $\mathbf{p}_{\mathrm{bsl}}$ has the maximum projection. It then appends the selected column vector $\left[\mathbf{A}_{\mathrm{can}}\right]_{:, k}$ to the RF precoder $\mathbf{P}_{\mathrm{RF}}$. The least squares solution to $\mathbf{p}_{\mathrm{BB}}$ is then calculated in step 9 and the contribution of the selected vector is removed in step 10. At this point, instead of loosing information by projecting $\left[\mathbf{A}_{\text {can }}\right]_{:, k}$ out as in the basic OMP approach, we implement, in steps 11-17, the previously described DDL mechanism which replaces the dictionary column $\left[\mathbf{A}_{\mathrm{can}}\right]_{:, k}$ with a 2-bit quantized vector close to the residual. The process continues until all $N_{\text {BS-RF }}$ beamforming vectors have been selected.

At the end of the $N_{\mathrm{BS}-\mathrm{RF}}$ iterations, the algorithm normalizes the digital baseband precoding vector $\mathbf{p}_{\mathrm{BB}}$ to satisfy the constraint $\left\|\mathbf{P}_{\mathrm{RF}} \mathbf{p}_{\mathrm{BB}}\right\|_{2}^{2}=1$ and outputs the constructed $M_{\mathrm{BS}} \times N_{\mathrm{RF}-\mathrm{BS}}$ $\mathrm{RF}$ precoding matrix $\mathbf{P}_{\mathrm{RF}}$.

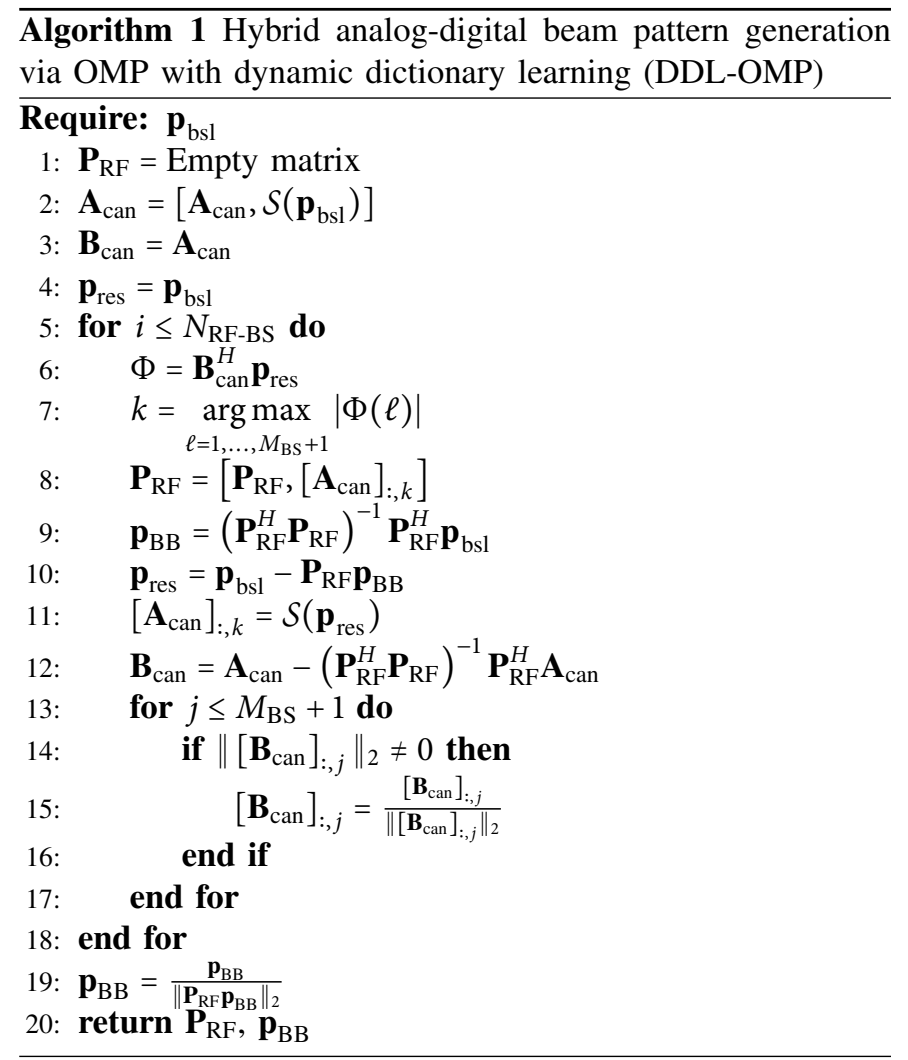

In the next section, we show how not only our DDL-OMP algorithm allows to synthesize beam patterns very close to the desired baseline ones, but it also significantly outperforms state-of-art approaches requiring higher complexity hardware.

\section{Simulation Results}

In this section, we first present simulation results to evaluate the ability of the DDL-OMP algorithm described in \$III-B to approximate the baseline fully-digital codebook design in \$II-A via a hybrid analog-digital beamforming architecture. 


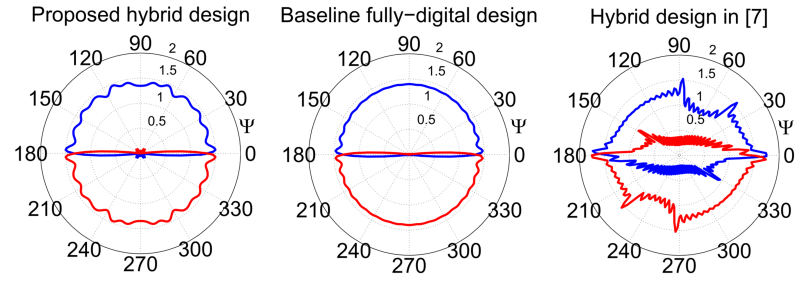

(a) Codebooks for stage 1

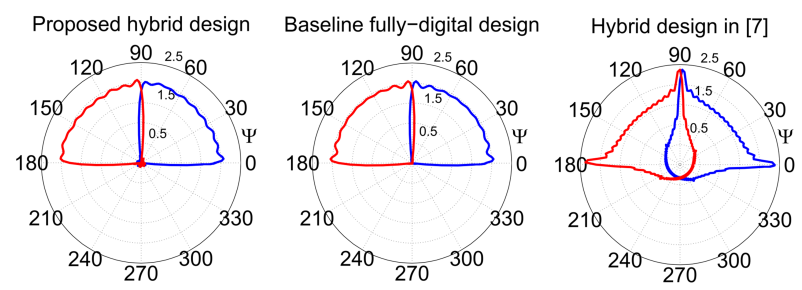

(b) Codebooks for stage 2

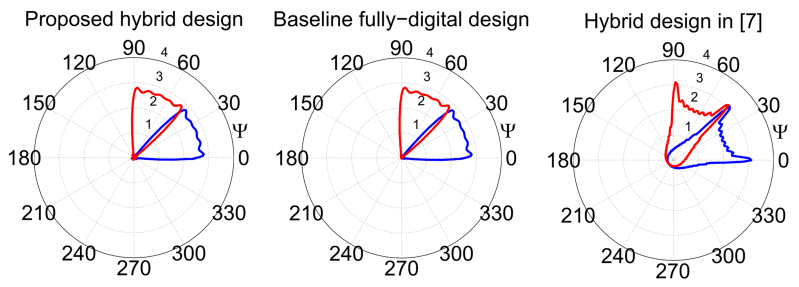

(c) Codebooks for stage 3

Fig. 4. Beam patterns for the first three stages of the beam training protocol: comparisons among the proposed algorithm, the solution presented in [7], and the ideal beamforming scheme based on FSM-KW with sidelobe attenuation $A=25 \mathrm{~dB}$

Second, the performance achieved by leveraging the designed hybrid codebooks for adaptive beam training are shown and compared with the literature.

\section{A. Beam patterns}

Examples of beam patterns resulting from applying the algorithm proposed in $\$$ III-B, the design presented in [7], and the baseline fully-digital beamforming scheme outlined in $\$$ III-A are plotted in Fig. 4 These patterns are generated by a BS having $M_{\mathrm{BS}}=64, \lambda / 2$-spaced isotropic antenna elements. The proposed hybrid analog-digital design exploits 2-bit RF phase shifters and $N_{\text {RF-BS }}=8 \mathrm{RF}$ chains while the hybrid codebooks in [7] are generated with $N_{\mathrm{RF}-\mathrm{BS}}=32 \mathrm{RF}$ chains and 7-bit RF phase shifters.

As evident from Fig. 4, the proposed algorithm provides almost optimal beam patterns with limited overlap between adjacent beams and excellent flatness over the covered sectors. Such qualitative evidence is quantitatively evaluated in Fig. 5 where the percentage of area common to two adjacent beams and the normalized peak-valley $(\mathrm{P}-\mathrm{V})$ value are plotted. The $\mathrm{P}-\mathrm{V}$ is a metric commonly adopted in optics to characterize the surface flatness of laser beams. It is calculated as the difference between the "highest" and "lowest" values on the sector normalized to the mean value and expressed in percentage terms. As shown, the proposed hybrid analog-digital beam patterns exhibit overlap and $\mathrm{P}-\mathrm{V}$ values close to those attained

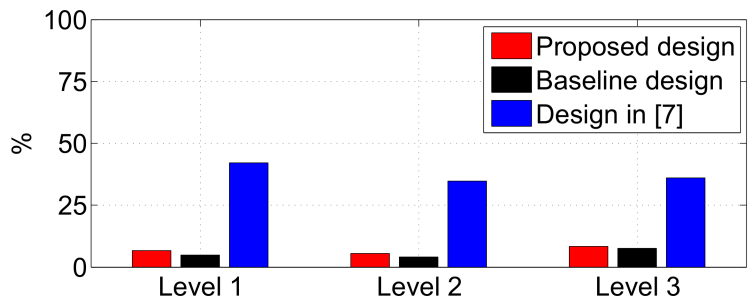

(a) Overlap between adjacent beams

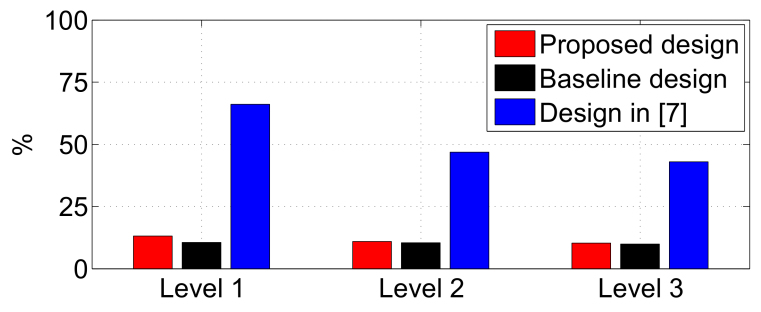

(b) Normalized peak-valley value (P-V)

Fig. 5. Evaluation of beam patterns in terms of (a) percentage of area that is common to two adjacent beams and (b) normalized peak-valley (P-V) value.

by the baseline fully-digital design. Compared to the solution proposed in [7], our approach displays roughly a fourfold performance improvement with reduced number of $R F$ chains and 2-bit $R F$ phase shifters.

\section{B. Beam training performance}

In a second set of simulations, we consider one BS with $M_{\mathrm{BS}}=64$ antennas and one MS with $M_{\mathrm{MS}}=32$, both featuring the hybrid analog-digital architecture in Fig. 1 and running the beam training algorithm described in $\$$ III The distance between the BS and MS is set to 50 meters and the path loss exponent of the propagation scenario is fixed to $n_{\text {ple }}=3$. The antenna arrays are ULAs with $\lambda / 2$-spaced isotropic radiators. We consider the geometric channel model described in Eq. 2 with a number of paths $L=3$. The AoDs/AoAs are assumed to take continuous values uniformly distributed in $[0,2 \pi]$. The transmit power at the BS is set to $30 \mathrm{dBm}$ and the system is assumed to operate at $28-\mathrm{GHz}$ carrier frequency with 100 $\mathrm{MHz}$ bandwidth.

The average absolute error on the estimation of the most powerful AoD and AoA for different BS/MS configurations is plotted in Fig. 6. Note that the BS is assumed to be equipped with a number of RF chains $N_{\text {RF-BS }}$ always greater than or equal to the number of RF chains $N_{\mathrm{RF}-\mathrm{MS}}$ at the MS. The numerical results are obtained from Monte Carlo simulations with 2000 independent channel realizations for each BS/MS configuration.

As evident from the graphs, the proposed hybrid analogdigital codebook design with 2-bit RF phase shifters provides AoD/AoA estimation errors always below $10^{\circ}$, independently from the number of RF chains at the BS and MS sides. Moreover, the overall performance is quite close to the baseline performance (dashed black line) of $6.5^{\circ}$ and $5.8^{\circ}$ respectively for the average AoD and AoA estimation errors achieved with 


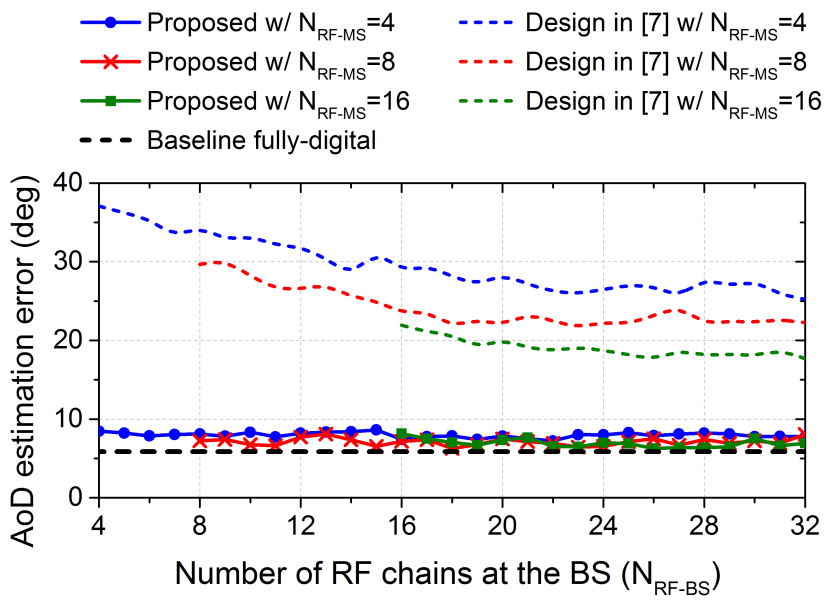

(a) AoD estimation

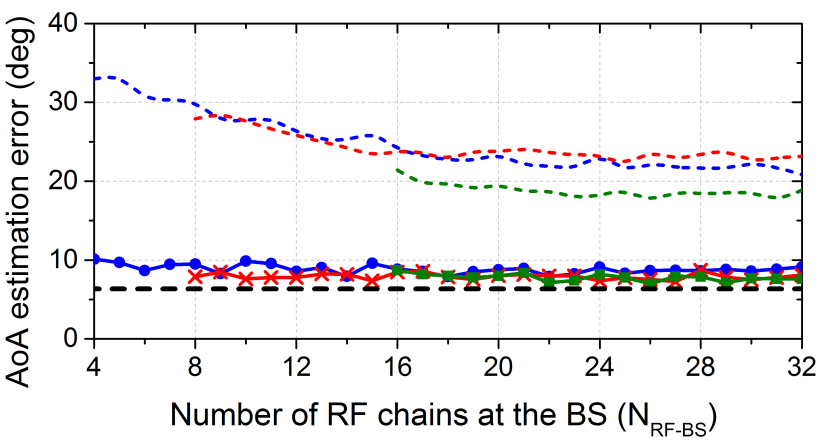

(b) AoA estimation

Fig. 6. Average absolute error in the estimation of the most powerful AoD (a) and AoA (b) using the beam training algorithm described in \$III Comparison among different transceiver architectures when varying the number of RF chains at the BS and MS sides.

BS and MS exploiting a fully-digital beamforming architecture based on FSM-KW codebooks.

As for the hybrid design proposed in [7] with 7-bit RF phase shifters, the minimum AoD/AoA estimation error is $17^{\circ}$ which is achieved with $N_{\mathrm{RF}-\mathrm{BS}}=26$ and $N_{\mathrm{RF}-\mathrm{MS}}=16 \mathrm{RF}$ chains at the BS and MS respectively. The average AoD/AoA estimation error is always above $22^{\circ}$ when the MS is equipped with less than $N_{\mathrm{RF}-\mathrm{MS}}=8 \mathrm{RF}$ chains.

Taken collectively, the presented results demonstrate that our algorithm is able to closely approximate a fully-digital beamformer by means of a very low complexity hybrid architecture. Besides, our algorithm outperforms one of the most relevant works in the literature [7], yet relying on lower complexity hardware. There are two fundamental reasons for this outcome. The first one, described in \$III-A is the adoption of the FSM-KW design as a reliable baseline codebook to shape almost ideal sector beam patterns with configurable beamwidth and steering direction. The second one, explained in \$III-B, is that the classical OMP algorithm adopted in [7] depletes its dictionary by nullifying some columns at each iteration. This translates into the use of a less informative dictionary as the algorithm progressively advances. On the contrary, the solution proposed in this paper provides superior performance thanks to the ability of the dictionary learning mechanism to dynamically and optimally update the OMP dictionary at each iteration.

\section{CONCLUSION}

In this paper, we proposed a practical codebook design for mmWave systems featuring hybrid analog-digital architecture with a number of RF chains much lower than the number of antenna elements and with 2-bit RF phase shifters. The results showed that our hybrid beamformer is able to shape beam patterns very close to those attained by a fully-digital architecture and to estimate the most promising AoA/AoD directions with performance close to the baseline accuracy.

\section{ACKNOWLEDGMENT}

The research leading to these results received funding from the European Commission H2020 programme under grant agreement no 671650 (5G PPP mmMAGIC project). This article is also partially supported by the Madrid Regional Government through the TIGRE5-CM program (S2013/ICE2919), the Ramon y Cajal grant from the Spanish Ministry of Economy and Competitiveness RYC-2012-10788, and the European Research Council grant ERC CoG 617721.

\section{REFERENCES}

[1] T. S. Rappaport et al., "Millimeter wave mobile communications for 5G cellular: It will work!" IEEE Access, vol. 1, pp. 335-349, 2013.

[2] mmMAGIC. Millimetre-Wave Based Mobile Radio Access Network for Fifth Generation Integrated Communications, EU 5G-PPP H2020ICT-2014-2 Project. [Online]. Available: http://5g-mmmagic.eu/

[3] A. Valdes-Garcia et al., "A SiGe BiCMOS 16-element phased-array transmitter for 60Ghz communications," in 2010 IEEE Solid-State Circuits Conference (ISSCC), Feb. 2010, pp. 218-219.

[4] Y. Tsang, A. Poon, and S. Addepalli, "Coding the beams: Improving beamforming training in mmwave communication system," in 2011 IEEE Global Telecommunications Conference (GLOBECOM), Houston, TX, USA, 2011, pp. 1-6.

[5] J. Song, J. Choi, and D. J. Love, "Codebook design for hybrid beamforming in millimeter wave systems," in 2015 IEEE International Conference on Communications (ICC), London, UK, Jun. 2015.

[6] O. E. Ayach, R. W. Heath, S. Abu-Surra, S. Rajagopal, and Z. Pi, "Low complexity precoding for large millimeter wave MIMO systems," in 2012 IEEE International Conference on Communications (ICC), Jun. 2012, pp. 3724-3729.

[7] A. Alkhateeb, O. E. Ayach, G. Leus, and R. W. Heath, "Channel estimation and hybrid precoding for millimeter wave cellular systems," IEEE Journal of Selected Topics in Signal Processing, vol. 8, no. 5, pp. 831-846, Oct. 2014.

[8] D. Pepe and D. Zito, "A novel phase shifter for $60 \mathrm{Ghz}$ phased arrays," in 2015 Irish Signals and Systems Conf. (ISSC), Jun. 2015, pp. 1-4.

[9] S. Payami, M. Shariat, M. Ghoraishi, and M. Dianati, "Effective RF codebook design and channel estimation for millimeter wave communication systems," in 2015 IEEE International Conference on Communication Workshop (ICCW), Jun. 2015, pp. 1226-1231.

[10] F. Sohrabi and Y. Wei, "Hybrid beamforming with finite-resolution phase shifters for large-scale MIMO systems," in 2015 IEEE 16th International Workshop on Signal Processing Advances in Wireless Communications (SPAWC), Jun. 2015, pp. 136-140.

[11] A. Alkhateeb, O. E. Ayach, G. Leus, and R. W. Heath, "Single-sided adaptive estimation of multi-path millimeter wave channels," in 2014 IEEE 15th International Workshop on Signal Processing Advances in Wireless Communications (SPAWC), Jun. 2014, pp. 125-129.

[12] S. J. Orfinidas. Electromagnetic waves and antennas. [Online]. Available: http://www.ece.rutgers.edu/ orfanidi/ewa/

[13] J. Wang et al., "Beamforming codebook design and performance evaluation for millimeter-wave WPAN," in 2009 IEEE 70th Vehicular Technology Conf. Fall (VTC Fall), 2009, pp. 1-6. 